IHEP 2000-12

\title{
Parametrizing and rephasing neutrino mixing
}

\author{
Yu. F. Pirogov \\ Institute for High Energy Physics, \\ Protvino, RU-142284 Moscow Region, Russia \\ Moscow Institute of Physics and Technology, \\ Dolgoprudny, Moscow Region, Russia
}

\begin{abstract}
Neutrino mixing in the standard model extensions, both renormalizable and effective, with arbitrary numbers of the singlet and lefthanded doublet neutrinos is investigated in a systematic fashion. The charged and neutral (the $Z$ and Higgs mediated) lepton currents are written under general Majorana condition, and the odservable independence of the choice of the condition, the rephasing invariance, is studied. A parametrization of the neutrino mixing matrices in the doublet-singlet factorized form is developed. Its relationship with the see-saw mechanism is shown in the limit of a small doublet-singlet mixing. The structure of the mixing matrices relevant to the neutrino oscillation experiments is explicated.
\end{abstract}

*E-mail: pirogov@mx.ihep.su 


\section{Introduction}

The lepton sector of the minimal Standard Model (SM) of electroweak interactions is amazingly simple and symmetric. Due to the absence of the right-handed neutrinos and neutrino masses, the SM predicts no flavour and $C P$ violation for leptons. Nevertheless, there is no known rule which would prohibit neutrinos from acquiring masses. More than this, there are numerous indications of the contrary. If so, the lepton mixing has to take place with all the subsequent phenomena such as flavour and $C P$ violation, neutrino oscillations, etc (as a recent review see, e.g., ref. [1]).

The lepton mixing, unlike the quark one, should generally be much more complicated. There are two main reasons for this. First, the number of the (iso)singlet neutrinos is a priori arbitrary relative to that of the (iso)doublet ones. Second, the Majorana masses for neutrinos are possible in addition to the Dirac ones. As a result, three types of associated problems arise. First, what is the total number of physical parameters, and how many of them are masses, mixing angles and $C P$ violating phases? Second, what do the lepton currents, both the vector and scalar ones, look like in terms of the mixing matrices? And third, how to parametrize the matrices explicitly? In the previous paper [2] (see also references therein) we have systematically studied the parameter counting problem for the SM extensions, both renormalizable and effective, with arbitrary numbers of the singlet and left-handed doublet neutrinos. Here we address ourselves to the second and third problems.

The gauge interactions of Majorana neutrinos for the SM extensions with arbitrary numbers of the singlet and left-handed doublet neutrinos were studied in ref. [3], where a parametrization of the neutrino mixing matrices was also proposed. The Yukawa neutrino interactions within the framework of the renormalizable SM extensions with an equal number of the singlet and doublet neutrinos were considered in ref. [4]. The studies of refs. [3, 4] were carried out traditionally under canonical Majorana condition. In the present paper these results are generalized under arbitrary Majorana condition for any SM extensions, both renormalizable and effective, with arbitrary numbers of the singlet and left-handed doublet neutrinos. The freedom of the choice of the Majorana condition, the rephasing invariance, is put as a corner- 
stone of the whole study. Some of our results are known in the literature in one form or another. Nevertheless, having being extended, they are hoped to be presented in the paper in a more systematic fashion.

In Section 2, the structure of the neutrino interactions, both gauge and Yukawa, are studied under arbitrary Majorana condition. In Section 3, the properties of the interactions under Majorana neutrino rephasing, including requirements for $C P$ invariance, are considered. A parametrization of the mixing matrices in the doublet-singlet factorized form is proposed in Section 4. Its relationship, under small doublet-singlet mixing, with the see-saw mechanism [5] is shown. And finally, the patterns of neutrino mixing matrices, relevant to neutrino oscillation experiments, are discussed in Section 5.

\section{Lagrangians and mixing matrices}

(i) Weak basis The most general renormalizable $S U(2)_{\mathrm{W}} \times U(1)_{\mathrm{Y}}$ invariant lepton Lagrangian of the SM extended by the right-handed neutrinos reads

$$
\begin{aligned}
\mathcal{L}= & \overline{l_{L}^{0}} i \not \supset l_{L}^{0}+\overline{e_{R}^{0}} i \not D e_{R}^{0}+\overline{\nu_{R}^{0}} i \not \partial \nu_{R}^{0} \\
& -\left(\overline{l_{L}^{0}} Y^{e} e_{R}^{0} \phi+\overline{l_{L}^{0}} Y^{\nu} \nu_{R}^{0} \phi^{C}+\frac{1}{2} \overline{\nu_{L}^{0 \mathrm{C}}} M^{\dagger} \nu_{R}^{0}+\text { h.c. }\right) .
\end{aligned}
$$

In eq. (四), the lepton doublet $l_{L}^{0}$ and singlet $e_{R}^{0}, \nu_{R}^{0}$ fields with a zero superscript mean those in a weak basis where, by definition, the symmetry properties are well stated. It is supposed that the ordinary chiral families of the SM with the doublet left-handed Weyl neutrinos in number $d \geq 3$ are added by the singlet (sterile) Weyl neutrinos in number $s \geq 0$. Let us designate such a renormalizable SM extensions as $(d, s)_{\mathrm{r}}$. A priori, one should retain $s$ and $d$ as arbitrary integers, both $s \leq d$ and $s>d$ being allowed. 1 Further, $\not D \equiv \gamma^{\alpha} D_{\alpha}$ is the generic covariant derivative which reduces to the ordinary one, $\not \partial=\gamma^{\alpha} \partial_{\alpha}$, for the hypercharge zero singlet neutrinos. Here

\footnotetext{
${ }^{1}$ We omit in the present analysis the possible vector-like lepton doublets. Hence, with account for the most probable exclusion of the fourth heavy chiral family [6], one should put in reality $d=3$. Nevertheless, we retain $d$ as a free parameter to better elucidate the parameter space structure of the extended SM.
} 
and in what follows the notations $\nu_{L}^{0 \mathrm{C}} \equiv\left(\nu_{R}^{0}\right)^{C}=C{\overline{\nu_{R}^{0}}}^{T}$, etc, are used for the particle-antiparticle conjugates of chiral fermions in the weak basis. $Y^{e}$ and $Y^{\nu}$ are the arbitrary complex $d \times d$ and $d \times s$ Yukawa matrices, respectively, and $M$ is a complex symmetric $s \times s$ matrix of the Majorana masses for the singlet neutrinos. Finally, $\phi$ is the Higgs isodoublet and $\phi^{C} \equiv i \tau_{2} \phi^{*}$ is its charge conjugate.

One can generalize the preceding considerations to the most exhaustive Dirac-Majorana case with the left-handed Majorana masses. The direct Majorana mass term for the doublet neutrinos is excluded in the minimal SM by the symmetry and renormalizability requirements. But in the extended SM as a low energy effective theory it could stem from the SM invariant operator of the fifth dimension

$$
-\mathcal{L}^{\prime}=\frac{1}{2 \Lambda}\left(\phi^{C \dagger} \tau_{i} \phi\right)\left(\overline{l_{R}^{0 C}} h i \tau_{2} \tau_{i} l_{L}^{0}\right)+\text { h.c. },
$$

with $\tau_{i}, i=1,2,3$ being the Pauli matrices, $h$ being a $d \times d$ symmetric constant matrix, $\Lambda \gg v$ being the lepton number violating mass scale (supposedly of order of the singlet Majorana masses) and $v$ being the Higgs vacuum expectation value. The above operator with the effective isotriplet field $\Delta_{i}=(1 / \Lambda)\left(\phi^{C \dagger} \tau_{i} \phi\right)$ reflects the oblique radiative corrections in the low energy Lagrangian produced by the physics beyond the SM. 2 With the Higgs doublet as

$$
\phi=\left(\begin{array}{c}
i w^{+} \\
\frac{1}{\sqrt{2}}(v+H+i z)
\end{array}\right)
$$

it yields (in the unitary gauge) the following mass and Yukawa term for neutrinos

$$
-\mathcal{L}^{\prime}=\frac{1}{2}\left(1+\frac{H}{v}\right)^{2} \overline{\nu_{R}^{0 C}} \mu \nu_{L}^{0}+\text { h.c. },
$$

where $\mu=h v^{2} / \Lambda$. Such an effective SM extension will be designated as $(d, s)$.

Now, let us introduce the complete one-handed neutrino collection (which can always be chosen, say, as left-handed)

$$
n_{L}^{0}=\left(\nu_{L}^{0}, \nu_{L}^{0 \mathrm{C}}\right)
$$

\footnotetext{
${ }^{2}$ Were the isotriplet $\Delta_{i}$ be considered as elementaty in the renormalizable framework, it would change only the emerging Yukawa interactions not affecting the mass and mixing matrices.
} 
so that $\left(n_{L}^{0}\right)^{\mathrm{C}} \equiv n_{R}^{0 \mathrm{C}}=\left(\nu_{R}^{0 \mathrm{C}}, \nu_{R}^{0}\right)$. In these notations, the total neutrino mass matrix $\mathcal{M}_{0}^{n}$ defined by the mass Lagrangian

$$
-\mathcal{L}_{\text {mass }}^{n}=\frac{1}{2} \overline{n_{R}^{0 \mathrm{C}}} \mathcal{M}_{0}^{n} n_{L}^{0}+\text { h.c. } \equiv-\frac{1}{2} n_{L}^{0 \mathrm{~T}} C^{-1} \mathcal{M}_{0}^{n} n_{L}^{0}+\text { h.c. }
$$

is clearly symmetric with account for $C^{T}=-C$. More particularly, it has the form

$$
\mathcal{M}_{0}^{n}=\left(\begin{array}{cc}
\mu & m \\
m^{T} & M
\end{array}\right),
$$

where $m \equiv Y^{*} v / \sqrt{2}$ is an arbitrary $d \times s$ matrix of the Dirac masses, $m^{T}$ is its transposed, $\mu$ and $M$ are, respectively, the $d \times d$ and $s \times s$ symmetric Majorana mass matrices from eqs. (11) and (4).

(ii) Mass basis Let us now consider the mass basis $n_{L}$ where, by definition, the neutrino mass matrix is diagonal. It is understood in this that the true neutrino mass eigenfields are $d+s$ four component fields $\mathcal{N}\left(n_{L}\right)$ bringing the neutrino kinetic Lagrangian to the diagonal positive form and simultaneously satisfying some subsidiary Majorana condition to halve the number of degrees of freedom. Such most general condition looks like [7]- [9]

$$
\mathcal{N}_{\varphi}^{C} \equiv \varphi \mathcal{N}_{\varphi}
$$

where $\mathcal{N}_{\varphi}^{C} \equiv C \overline{\mathcal{N}}_{\varphi}^{T}$ and $\varphi=\operatorname{diag}\left(\varphi_{1}, \ldots, \varphi_{d+s}\right)$ is a diagonal phase matrix. Here and in what follows we use the notations with subscript $\varphi$ to stress that quantity at hand generally depends on $\varphi$. 3 Expressing $\mathcal{N}_{\varphi}$ through Weyl spinors as $\mathcal{N}_{\varphi}=\varphi_{L} n_{L} \oplus \varphi_{R} n_{R}^{C}$ with some diagonal phase matrices $\varphi_{L}$ and $\varphi_{R}$, one finds the Majorana condition to fulfil if $\varphi_{L} \varphi_{R}=\varphi^{*}$. Without loss of generality one can put, e.g., $\varphi_{L}=I, \varphi_{R}=\varphi^{*}$, so that 1

$$
\mathcal{N}_{\varphi}=n_{L} \oplus \varphi^{*} n_{R}^{\mathrm{C}}
$$

This choice is advantageous because it results in the simplest form for the charged current which is left-handed (see later on).

\footnotetext{
${ }^{3}$ Note that the maximum number of the independent Majorana specific phases in $\varphi$ might be $d+s-1$ because an overall neutrino phase is unobservable.

${ }^{4}$ Note that eqs. (8), (9) do not put any constraint on the original Weyl fields $n_{L}$.
} 
In these terms, we demand the kinetic part of the neutrino Lagrangian be

$$
\mathcal{L}_{\text {kin }}^{n}=\frac{1}{2} \overline{\mathcal{N}}_{\varphi} i \not \partial \mathcal{N}_{\varphi}-\frac{1}{2} \overline{\mathcal{N}}_{\varphi} \mathcal{M}_{\mathrm{diag}}^{n} \mathcal{N}_{\varphi}
$$

with a non-negative diagonal mass matrix $\mathcal{M}_{\text {diag }}^{n}$ idependent of $\varphi$. To this end, let us choose the $(d+s) \times(d+s)$ unitary transformation $\mathcal{U}_{\varphi}^{n}$

$$
n_{L}^{0}=\mathcal{U}_{\varphi}^{n} n_{L}
$$

so that

$$
\mathcal{U}_{\varphi}^{n T} \mathcal{M}_{0}^{n} \mathcal{U}_{\varphi}^{n}=\varphi \mathcal{M}_{\text {diag }}^{n}
$$

with

$$
\mathcal{M}_{\text {diag }}^{n}=\operatorname{diag}\left(m_{1}^{\nu}, \ldots m_{d}^{\nu} ; M_{1}^{N}, \ldots M_{s}^{N}\right) .
$$

With account for $\overline{\mathcal{N}}_{\varphi}=\overline{\mathcal{N}}_{\varphi}^{C} \varphi \equiv-\mathcal{N}_{\varphi}^{T} C^{-1} \varphi$, the neutrino kinetic Lagrangian takes the required form of eq. (10). At $s \leq d$ for the $(d, s)_{\text {r }}$ extension, $d-s$ elements $m^{\nu}$ are zero. This reflects the fact that in this case the rank of the $(d+s) \times(d+s)$ matrix given by eq. (7) with $\mu=0$ is $2 s$. At $s>d$ the rank of the matrix is generally $d+s$ and hence there is no massless neutrinos.

Similarly, the charged lepton fields $e_{\chi}(\chi=L, R)$ in the mass basis are defined as

$$
e_{\chi}^{0}=\mathcal{U}_{\chi}^{e} e_{\chi}
$$

with the unitary $d \times d$ matrices $\mathcal{U}_{\chi}^{e}$, so that the bi-diagonalization of relevant mass matrix looks like

$$
\mathcal{U}_{L}^{e \dagger} \mathcal{M}_{0}^{e} \mathcal{U}_{R}^{e}=\mathcal{M}_{\text {diag }}^{e}=\operatorname{diag}\left(m_{1}^{e}, \ldots, m_{d}^{e}\right)
$$

By means of the global symmetries of the kinetic part of Lagrangian (11) one can arrange, without loss of generality, the charged lepton weak basis to coincide with the mass one. This means that $\mathcal{M}_{0}^{e}$ can be chosen diagonal ab initio, so that $\mathcal{U}_{L}^{e}=\mathcal{U}_{R}^{e}=I$. The associated neutrinos are usually referred to as the flavour ones.6 Traditionally, the corresponding basis is used when

\footnotetext{
${ }^{5}$ This notations correspond to partition $\mathcal{N}_{\varphi} \equiv(\nu, N)_{\varphi}$ and tacitly imply the see-saw hierarchy $m^{\nu} \ll M^{N}$ for all the elements, with $\nu$ being (quasi-)doublet neutrinos and $N$ being (quasi-)singlet ones. Nevertheless there might be experimental indications of the existence of at least one light singlet neutrino [1].

${ }^{6}$ Unfortunatelly, this is unlike the quark sector where flavour is synonymous with the mass eigenstate.
} 
discussing the neutrino oscillation phenomenon. For simplicity, it is adopted in what follows. But in fact there is no need for such a particular choice. Moreover, the mass basis suffices to describe the neutrino oscillations without resort to the weak eigenstates 10.0

Now, the charged current Lagrangian in the mass basis reads 8

$$
-\mathcal{L}_{W}=\frac{g}{\sqrt{2}} W_{\alpha}^{-} \overline{e_{L}} \gamma^{\alpha} V_{\varphi} \mathcal{N}_{\varphi L}+\text { h.c. }
$$

where the rectangular $d \times(d+s)$ mixing matrix for the charged currents is

$$
V_{\varphi}=\mathcal{U}_{L}^{e \dagger} P^{e n} \mathcal{U}_{\varphi}^{n}
$$

with the charged current matrix in the weak basis given by

$$
P^{e n}=\left(\begin{array}{cc}
I_{d} & O_{d \times s}
\end{array}\right)
$$

$I_{d}$ being the $d$-dimensional identity matrix and $O_{d \times s}$ being $d \times s$ zero matrix. The lepton mixing matrix $V_{\varphi}$ is a counterpart of the quark CKM matrix. It follows from eqs. (17) and (18) that

$$
V_{\varphi} V_{\varphi}^{\dagger}=I_{d}
$$

though $V_{\varphi}^{\dagger} V_{\varphi} \neq I_{d+s}$. Eq. (19) can be regarded as the one-sided unitarity condition at $s \neq 0$.

The neutral current Lagrangian with the SM neutral current operator $T_{3}-s_{W}^{2} Q$ in the mass basis is as follows:

$$
-\mathcal{L}_{Z}=\frac{g}{c_{W}} Z_{\alpha}\left(-\frac{1}{2} \overline{e_{L}} \gamma^{\alpha} e_{L}+s_{W}^{2} \bar{e} \gamma^{\alpha} e+\frac{1}{2} \overline{\mathcal{N}}_{\varphi L} \gamma^{\alpha} X_{\varphi} \mathcal{N}_{\varphi L}\right),
$$

where the $(d+s) \times(d+s)$ neutrino mixing matrix for the neutral currents is

$$
X_{\varphi}=\mathcal{U}_{\varphi}^{n \dagger} P^{n} \mathcal{U}_{\varphi}^{n}
$$

\footnotetext{
${ }^{7}$ When there is an admixture of the heavy Majorana neutrinos, it is only the coherent part of the light neutrinos what has the meaning of a flavour state. Note that so modified flavour states are non-orthogonal and process dependent.

${ }^{8}$ Note that due to the supposed absence of the vector-like lepton doublets, the righthanded charged currents do not emerge.
} 
with the on-doublet neutrino projector $\left(P^{n 2}=P^{n}\right)$

$$
P^{n}=\operatorname{diag}(\underbrace{1, \ldots, 1}_{d} ; \underbrace{0, \ldots, 0}_{s}) .
$$

Here one puts $c_{W} \equiv \cos \theta_{W}, s_{W} \equiv \sin \theta_{W}$ with $\theta_{W}$ being the Weinberg angle. Clearly, $X_{\varphi}$ is a Hermitian projective matrix: $X_{\varphi}=X_{\varphi}^{\dagger}, X_{\varphi}^{2}=X_{\varphi} \neq I$. Due to eqs. (17), (18) the relation

$$
X_{\varphi}=V_{\varphi}^{\dagger} V_{\varphi}
$$

between the neutral and charged current mixing matrices is obeyed. For the $(d, 0)$ extension one has $P^{n}=I_{d}$, so that $X_{\varphi} \equiv I_{d}$ and thus the $d \times d$ matrix $V_{\varphi}$ is unitary. More than this, for the renormalizable $(d, 0)_{\mathrm{r}}$ extensions one can always put $\mathcal{U}_{\varphi}^{n}=I$, so that $V_{\varphi}=I_{d}$ also follows. Hence, the lepton flavour conservation of the minimal SM with the residual symmetry $U(1)^{d}$ is readily recovered.

For the renormalizable extensions $(d, s)_{\mathrm{r}}$, the Yukawa Lagrangian looks like

$$
\begin{aligned}
-\mathcal{L}_{Y}= & \frac{H}{v} \bar{e} \mathcal{M}_{\mathrm{diag}}^{e} e+\frac{z}{v} \bar{e} \mathcal{M}_{\mathrm{diag}}^{e} i \gamma_{5} e \\
+ & \left(\frac{1}{2} \frac{H+i z}{v} \overline{\mathcal{N}}_{\varphi R}\left(\varphi^{*} X_{\varphi}^{T} \varphi \mathcal{M}_{\mathrm{diag}}^{n}+\mathcal{M}_{\mathrm{diag}}^{n} X_{\varphi}\right) \mathcal{N}_{\varphi L}\right. \\
& \left.+\sqrt{2} i \frac{w^{-}}{v}\left(\overline{e_{L}} V_{\varphi} \mathcal{M}_{\mathrm{diag}}^{n} \mathcal{N}_{\varphi R}-\overline{e_{R}} \mathcal{M}_{\mathrm{diag}}^{e} V_{\varphi} \mathcal{N}_{\varphi L}\right)+\text { h.c. }\right) .
\end{aligned}
$$

Here use is made of the constraint

$$
X_{\varphi}^{T} \varphi \mathcal{M}_{\mathrm{diag}}^{n} X_{\varphi}=0,
$$

which follows from a more particular one

$$
P^{n} \mathcal{U}_{\varphi}^{n *} \varphi \mathcal{M}_{\text {diag }}^{n} \mathcal{U}_{\varphi}^{n \dagger} P^{n}=0
$$

and reflects the absence of the $d \times d$ symmetric left-handed Majorana mass term $\mu$ in eq. (7).

For the general extensions $(d, s)$, the constraint eq. (25) should be dropped off. This results in addition of a number of interaction terms to Yukawa Lagrangian. E.g., according to eq. (4) one should add in the unitary gauge the term

$$
-\mathcal{L}_{Y}^{\prime}=\frac{1}{2}\left(\frac{H}{v}\right)^{2} \overline{\mathcal{N}}_{\varphi R} \varphi^{*} X_{\varphi}^{T} \varphi \mathcal{M}_{\text {diag }}^{n} X_{\varphi} \mathcal{N}_{\varphi L}+\text { h.c. },
$$

the linear in $H$ term being cancelled by a similar one present now in $\mathcal{L}_{Y}$. 


\section{Rephasing invariance}

Consider the group of transformations consisting of the Majorana field rephasing $\mathcal{N}_{\varphi} \rightarrow \Phi^{1 / 2} \mathcal{N}_{\varphi}$ followed by transformations

$$
\begin{aligned}
\varphi & \rightarrow \varphi \Phi^{*} \\
V_{\varphi} & \rightarrow V_{\varphi} \Phi^{* 1 / 2}
\end{aligned}
$$

with a diagonal phase matrix $\Phi=\operatorname{diag}\left(\Phi_{1}, \ldots, \Phi_{d+s}\right)$. As a result, one also gets $X_{\varphi} \rightarrow \Phi^{1 / 2} X_{\varphi} \Phi^{* 1 / 2}$. All the Lagrangians are clearly rephasing invariant. It follows from eq. (28) that independent rephasing invariant quantities containing $\varphi$ may be chosen as $V_{\varphi} \varphi^{* 1 / 2}\left(\varphi^{1 / 2} X_{\varphi} \varphi^{* 1 / 2}\right)$ and $\varphi^{1 / 2} \mathcal{N}_{\varphi}$. Thus, the rephasing allows one to extract a number of neutrino phases from $V_{\varphi}$ and to reabsorb them in $\varphi$ (or v.v.). Observables depend only on the sum of the complementary phases of $V_{\varphi}$ and $\varphi^{* 1 / 2}$ as well as of $\mathcal{N}_{\varphi}$ and $\varphi^{1 / 2}$, but not separately on each of them (in addition to phases in the rephasing invariant combinations of the matrix $V_{\varphi}$ itself). Clearly, it is not a particular choice of the Majorana condition but the invariance with respect to this choice which is physically meaningful.

The rephasing invariance permits one to choose $\varphi$ most appropriate to the problem at hand. The reason is that only the Higgs vertices and the neutrino wave functions (and thus the $\left\langle\mathcal{N}_{\varphi} \overline{\mathcal{N}_{\varphi}^{C}}>\right.$ propagators) depend explicitly on $\varphi$, whereas the gauge vertices and the $\left\langle\mathcal{N}_{\varphi} \overline{\mathcal{N}_{\varphi}}\right\rangle$ propagators do not depend on it. As a result, if the matrix element for a particular process does not contain $\varphi$ explicitly one can extract by means of the rephasing as many Majorana specific phases from $V_{\varphi}$ as possible. The rephasing invariance then insures that under other $\varphi$ these phases, though being superficially present in $V_{\varphi}$, would not enter nevertheless the final results.

\footnotetext{
${ }^{9}$ Stress that due to rephasing invariance, fixing a choice for $\varphi$ has nothing to do with the real physical properties of the Majorana neutrinos, in particular with those concerning $C$ conjugation. The last properties are described additionally by the fact that if the neutrino mass eigenstates do possess definite $C$ parity $\eta_{C}=\operatorname{diag}( \pm 1)$, then the $C$ conjugation for the Majorana eigenfields shoud be consistently redefined [8] as $\mathcal{N}_{\varphi} \stackrel{C}{\rightarrow} \mathcal{N}_{\varphi}^{C_{\varphi}} \equiv \eta_{C} \varphi^{*} \mathcal{N}_{\varphi}^{C}$, where traditionally $\mathcal{N}_{\varphi}^{C} \equiv C \overline{\mathcal{N}}_{\varphi}^{T}$. It follows that the modified (anti-)self-charge conjugacy condition $\mathcal{N}_{\varphi}^{C_{\varphi}}=\eta_{C} \mathcal{N}_{\varphi}$ is indeed satisfied independent of $\varphi$. Nevertheless, attempts are sometimes made in the literature to ascribe physics content to the Majorana condition being chosen superficially in the self- or anti-self-charge conjugate form. The emerging results are thus misleading.
} 
To illustrate, the amplitude for the (chirality conserving) $\mathcal{N} \mathcal{N}$ oscillations

$$
\mathcal{A}_{0}(t)=V_{\varphi} e^{-i E t} V_{\varphi}^{\dagger}
$$

clearly does not depend on the Majorana specific phases capable of being stored in $\varphi$, whereas amplitude for the (chirality flipping) $\mathcal{N N}^{C}$ oscillations

$$
\mathcal{A}_{1}(t)=V_{\varphi} e^{-i E t} \varphi^{*} \mathcal{M}_{\mathrm{diag}}^{n} E^{-1} V_{\varphi}^{T}
$$

does depend on the phases. In the above, $E$ is the diagonal energy matrix for the light neutrinos. The same is true for the neutrino mass elements $\nu \nu^{C}$ in the weak basis

$$
\mathcal{M}_{\nu_{e} \nu_{e^{\prime}}}^{n *}=\left(V_{\varphi} \varphi^{*} \mathcal{M}_{\text {diag }}^{n} V_{\varphi}^{T}\right)_{\nu_{e} \nu_{e^{\prime}}},
$$

which determine the rates of the neutrinoless double $\beta$-decay (at $e^{\prime}=e$ ) or $e \bar{\mu}$ conversion $\left(\right.$ at $\left.e^{\prime}=\mu\right) .10$

(i) Canonical Majorana condition Sometimes it might be tempting to go to a basis where the Majorana neutrino wave functions have a canonical form. Namely, the rephasing by $\Phi=\varphi$ yields $\varphi \rightarrow I$, with $I$ being unity matrix, and transformed fields $\mathcal{N}_{I}$ satisfy the canonical Majorana condition $\mathcal{N}_{I}^{C}=\mathcal{N}_{I}$. Under this condition, all the physical mixing parameters reside only in mixing matrices. With account for the $X_{\varphi}$ Hermiticity property $\mathcal{R} e X_{\varphi}^{T}=\mathcal{R} e X_{\varphi}$ and $\mathcal{I} m X_{\varphi}^{T}=-\mathcal{I} m X_{\varphi}$, the neutrino neutral current parts of Lagrangians (20) and (24) can now be re-expressed in a simpler form

$$
-\mathcal{L}_{Z}^{n}=\frac{g}{4 c_{W}} Z_{\alpha} \overline{\mathcal{N}}_{I} \gamma^{\alpha}\left(i \mathcal{I} m X_{I}-\gamma_{5} \mathcal{R} \text { e } X_{I}\right) \mathcal{N}_{I}
$$

and 11

$$
\begin{aligned}
-\mathcal{L}_{Y}^{n}= & \frac{1}{2} \frac{H}{v} \overline{\mathcal{N}}_{I}\left(\mathcal{R} e X_{I}+i \gamma_{5} \mathcal{I} m X_{I}\right) \mathcal{M}_{\text {diag }}^{n} \mathcal{N}_{I} \\
& +\frac{1}{2} \frac{z}{v} \overline{\mathcal{N}}_{I}\left(\mathcal{I} m X_{I}-i \gamma_{5} \operatorname{Re} X_{I}\right) \mathcal{M}_{\text {diag }}^{n} \mathcal{N}_{I}+\text { h.c. }
\end{aligned}
$$

\footnotetext{
${ }^{10}$ Note that according to eqs. (24) and (27) the (chirality flipping) Yukawa interactions might also serve as a probe of the Majorana specific phases.

${ }^{11}$ Under $\varphi=I$, the Yukawa term for the renormalizable extensions $(n, n)_{\mathrm{r}}$ was found in ref. [四.
} 
(ii) $C P$ invariance It is well known that for a field theory not to explicitly violate $C P$ there should be allowed a weak basis where all the parameters in the Lagrangian are real. Under this condition, the neutrino mass matrix $\mathcal{M}^{n}$, being symmetric, can always be brought to the (real) diagonal form (generally, not positive definite) by means of an orthogonal transformation $\mathcal{U}^{n}=\mathcal{O}^{n}$ with the effect

$$
\mathcal{O}^{n T} \mathcal{M}^{n} \mathcal{O}^{n}=\eta_{M} \mathcal{M}_{\text {diag }}^{n} .
$$

Here $\eta_{M} \equiv \operatorname{diag}( \pm 1)$ is the mass signature matrix which is completely determined by the original $\mathcal{M}^{n}$. Clearly, the mixing matrix $V_{\eta_{M}} \equiv \mathcal{R}=P^{e n} \mathcal{O}^{n}$ is real.12 In the rephasing invariant form one gets $V_{\varphi}=\mathcal{R}\left(\varphi \eta_{M}\right)^{1 / 2}$, and hence the condition for $C P$ invariance looks like

$$
V_{\varphi}=V_{\varphi}^{*} \varphi \eta_{M}
$$

as well as

$$
X_{\varphi}=\eta_{M} \varphi^{*} X_{\varphi}^{*} \varphi \eta_{M} .
$$

Stress that CP conservation does not mean $V_{\varphi}$ and $X_{\varphi}$ to be real in general.

In the mass basis, one can define the $C P$ conjugation (in the unitary gauge) as

$$
\begin{aligned}
e(x) & \rightarrow \gamma_{0} e^{C}\left(x^{P}\right), \\
\mathcal{N}_{\varphi}(x) & \rightarrow \eta_{C P} \varphi^{*} \gamma_{0} \mathcal{N}_{\varphi}^{C}\left(x^{P}\right), \\
W^{ \pm}(x) & \rightarrow-W^{\mp P}\left(x^{P}\right), \\
Z(x) & \rightarrow-Z^{P}\left(x^{P}\right), \\
H(x) & \rightarrow H\left(x^{P}\right)
\end{aligned}
$$

(with $x^{P} \equiv\left(x_{0},-\vec{x}\right)$, etc). The definition is clearly rephasing invariant. Here $i \eta_{C P}$, with $\eta_{C P} \equiv \operatorname{diag}( \pm 1)$, is the matrix of the (relative) $C P$ parities for the neutrino mass eigenstates [8, 9]. In the above, $\eta_{C P}$ is not arbitrary but is to be properly defined for consistency. Namely, under eq. (37) the whole Lagrangian can be shown to transform into itself where substitutions

$$
\begin{aligned}
\varphi & \rightarrow \varphi, \\
V_{\varphi} & \rightarrow V_{\varphi}^{*} \varphi \eta_{C P}
\end{aligned}
$$

\footnotetext{
${ }^{12}$ Here and in what follows, the basis where $\mathcal{U}_{L}^{e}=\mathcal{U}_{R}^{e}=I$ is generally chosen for simplicity.
} 
are made. Imposing the requirement of $C P$ invariance one arrives with account for eq. (35) at the identity

$$
\eta_{C P} \equiv \eta_{M}
$$

This identity insures the consistency of the description of $C P$ invariance directly in terms of the rephasing invariant quantities, which being built of $V_{\varphi}$ and $\varphi$ depend on $\eta_{M}$, with the description in terms of the explicit $C P$ transformations eq. (37) being dependent on $\eta_{C P}$.

In particular, in the case of $\mathrm{CP}$ conservation one gets for the amplitudes of eqs. (29)-(31)

$$
\begin{aligned}
\mathcal{A}_{0}(t) & =V_{\varphi} e^{-i E t} \eta_{C P} \varphi^{*} V_{\varphi}^{T} \\
\mathcal{A}_{1}(t) & =V_{\varphi} e^{-i E t} \eta_{C P} \mathcal{M}_{\mathrm{diag}}^{n} E^{-1} V_{\varphi}^{\dagger} \\
\mathcal{M}_{\nu_{e} \nu_{e^{\prime}}}^{n *} & =\left(V_{\varphi} \eta_{C P} \mathcal{M}_{\mathrm{diag}}^{n} V_{\varphi}^{\dagger}\right)_{\nu_{e} \nu_{e^{\prime}}} .
\end{aligned}
$$

At $\nu_{e}=\nu_{e^{\prime}}$, the last line explicitly demonstrates the possibility for the (partial) compensation of various contributions to the lepton number violating $e \bar{e}$ transition under $C P$ conservation. At $\varphi=\eta_{C P}$, the matrix $V_{\varphi}$ (as well as $\left.X_{\varphi}\right)$ becomes pure real, $V_{\eta_{C P}} \equiv \mathcal{R}$, so that

$$
\begin{aligned}
\mathcal{A}_{0}(t) & =\mathcal{R} e^{-i E t} \mathcal{R}^{T} \\
\mathcal{A}_{1}(t) & =\mathcal{R} e^{-i E t} \eta_{C P} \mathcal{M}_{\mathrm{diag}}^{n} E^{-1} \mathcal{R}^{T} \\
\mathcal{M}_{\nu_{e} \nu_{e^{\prime}}}^{n} & =\left(\mathcal{R} \eta_{C P} \mathcal{M}_{\mathrm{diag}}^{n} \mathcal{R}^{T}\right)_{\nu_{e} \nu_{e^{\prime}}} .
\end{aligned}
$$

The basis $\mathcal{N}_{\eta_{C P}}$ may be called as the $C P$-associated one. In a sense, it might present the most natural choice for the $C P$ conserving theory, all other bases being equivalent though probably less convenient. Thus, under canonical Majorana condition $\varphi=I$ the elements of $V_{I}$ (and $X_{I}$ ) in the $C P$ conserving theory should be according to eq. (35) either pure real or imaginary [8, 9, 11, and this has nothing to do with the maximal $C P$ violation as it might superficially seem.

\section{Doublet-singlet parametrization}

(i) General case A mathematical parametrization of the neutrino mixing matrix $\mathcal{U}^{n}$ is given in ref. [3]. An alternative physical prescription, heavily 
relying on the doublet-singlet neutrino content and thus being useful for practical purposes, is proposed in the present paper. For simplicity, the subscript $\varphi$ will be omitted in what follows. First of all note that by means of the global symmetries one can always achieve, without loss of generality, that $\mathcal{U}_{L}^{e}=\mathcal{U}_{R}^{e}=I$. 13 Now, before applying any restrictions on the neutrino mass matrix $\mathcal{M}^{n}$ the $(d+s) \times(d+s)$ unitary mixing matrix $\mathcal{U}^{n}$ is arbitrary and can be decomposed in a unique way (at least in a neighborhood of unity) as

$$
\mathcal{U}^{n}=\mathcal{U}_{d}^{n} \mathcal{U}_{s}^{n} \mathcal{U}_{m}^{n}
$$

Here $\mathcal{U}_{d}^{n}$ is a unitary $d \times d$ matrix in the doublet neutrino subspace corresponding to indices $f=1, \ldots, d$. This matrix is spanned on $d^{2}$ generators and depends on $d(d-1) / 2$ mixing angles and $d(d+1) / 2$ phases. More particularly, one can put

$$
\mathcal{U}_{d}^{n}=\left(\begin{array}{cc}
U_{d}^{\nu} & 0 \\
0 & I_{s}
\end{array}\right)
$$

with a $d \times d$ unitary matrix $U_{d}^{\nu}$. There is still a freedom of $d$ charged lepton phase redefinition which is left after the mass matrix in eq. (15) is diagonalized. According to eqs. (17) and (42) this freedom can be used to eliminate $d$ phases out of $\mathcal{U}_{d}^{n}$. It clearly leaves only $d(d-1) / 2$ independent phases in this matrix (and equal number of mixing angles).

Now, one can write down the following explicit parametrization for $U_{d}$ $(d>1)$ in terms of the modified Pontryagin's coordinates of the second kind [3]

$$
U_{d}^{\nu}=u_{\operatorname{diag}}(\alpha) \prod_{\substack{f, g=1, \ldots, d \\ f<g}} \otimes u_{f g}(\theta, \delta) .
$$

The product above should be understood in some particular (but a priori unspecified) order. Here $u_{\text {diag }}$ is a diagonal $d \times d$ phase matrix $u_{\text {diag }}(\alpha)=$ $\operatorname{diag}\left(e^{i \alpha_{1}}, \ldots, e^{i \alpha_{d}}\right)$ which differs equivalent parametrizations and is at our disposal. (At $d=1$, one has $U_{1}^{\nu}=e^{i \alpha_{1}}$.) A basic matrix $u_{f g}$ ("complex rotation"), one of a set of $d(d-1) / 2$ unitary $S U(2)$ submatrices, acts in the $f g$ plane, $f \neq g$, and depends only on one mixing angle $\theta_{f g}$ and one phase $\delta_{f g}$

$$
u_{f g}=\exp \left(\begin{array}{cc}
0 & \theta_{f g} e^{i \delta_{f g}} \\
-\theta_{f g} e^{-i \delta_{f g}} & 0
\end{array}\right)=\left(\begin{array}{cc}
\cos \theta_{f g} & \sin \theta_{f g} e^{i \delta_{f g}} \\
-\sin \theta_{f g} e^{-i \delta_{f g}} & \cos \theta_{f g}
\end{array}\right) .
$$

\footnotetext{
${ }^{13}$ For this reason, lepton mixing is synonimous with the neutrino one.
} 
By means of the identity

$$
u_{\text {diag }}(\alpha) u_{f g}\left(\theta_{f g}, \delta_{f g}\right) u_{\text {diag }}^{\dagger}(\alpha)=u_{f g}\left(\theta_{f g}, \alpha_{f}+\delta_{f g}-\alpha_{g}\right)
$$

one can eliminate $d-1 \delta$ 's out of $U_{d}^{\nu}$ and to transform these phases into the same number of the Majorana specific ones, the $d$-th of the last phases being unphysical.14 It clearly leaves $(d-1)(d-2) / 2$ CKM-like phases and $d-1$ Majorana specific ones. Thus, under proper phase redefinitions the matrix $U_{d}^{\nu}$ may be chosen in experimentally viable cases $d=2$ and 3 , respectively, as

$$
U_{2}^{\nu}=\left(\begin{array}{cc}
c & s \\
-s & c
\end{array}\right)\left(\begin{array}{cc}
e^{i \gamma} & 0 \\
0 & 1
\end{array}\right)
$$

and

$$
\begin{aligned}
U_{3}^{\nu} & =\left(\begin{array}{ccc}
c_{3} & s_{3} & 0 \\
-s_{3} & c_{3} & 0 \\
0 & 0 & 1
\end{array}\right)\left(\begin{array}{ccc}
c_{2} & 0 & s_{2} \\
0 & 1 & 0 \\
-s_{2} & 0 & c_{2}
\end{array}\right) \\
& \times\left(\begin{array}{ccc}
1 & 0 & 0 \\
0 & c_{1} & s_{1} e^{i \delta} \\
0 & -s_{1} e^{-i \delta} & c_{1}
\end{array}\right)\left(\begin{array}{ccc}
e^{i \gamma_{1}} & 0 & 0 \\
0 & e^{i \gamma_{2}} & 0 \\
0 & 0 & 1
\end{array}\right),
\end{aligned}
$$

where $c \equiv \cos \theta$ and $s \equiv \sin \theta$. Clearly, one can shift the ordinary phase $\delta$ to any of the $s_{i}, i=1,2,3$.

Further, $\mathcal{U}_{s}^{n}$ is the counterpart of $\mathcal{U}_{d}^{n}$ in the singlet neutrino subspace with indices $f=d+1, \ldots, d+s$, being spanned on the $s^{2}$ generators and dependent on $s(s-1) / 2$ mixing angles and $s(s+1) / 2$ phases. One has

$$
\mathcal{U}_{s}^{n}=\left(\begin{array}{cc}
I_{d} & 0 \\
0 & U_{s}^{N}
\end{array}\right)
$$

with a $s \times s$ unitary matrix $U_{s}^{N}$. Clearly, $\mathcal{U}_{d}^{n}$ and $\mathcal{U}_{s}^{n}$ commutes with each other. According to eq. (17) the matrix $\mathcal{U}_{s}^{n}$ is irrelevant for observables. Hence, by means of the global symmetries one can always achieve, without loss of generality, that $U_{s}^{N}=\operatorname{diag}\left(e^{i \alpha_{d+1}}, \ldots, e^{i \alpha_{d+s}}\right)$, with $\alpha$ 's being at our disposal. This choice is advantageous to subsequently expose the Majorana specific phases in $\mathcal{U}^{n}$.

\footnotetext{
${ }^{14}$ Strictly speaking, this is true only for the $(d, 0)$ case. For the $(d, s)$ extension the Majorana specific phases could be exposed only after taking into account the matrix $\mathcal{U}_{m}^{n}$.
} 
Finally, $\mathcal{U}_{m}^{n}$ is a unitary $(d+s) \times(d+s)$ matrix spanned on $2 s d$ generators which mix the two subspaces 15 This matrix depends generally on $s d$ mixing angles and the same number of phases. It follows from eqs. (21) and (42) that the neutral current mixing matrix takes the form

$$
X=\mathcal{U}_{m}^{n \dagger} P^{n} \mathcal{U}_{m}^{n}
$$

In other words, it depends entirely on the parameters of $\mathcal{U}_{m}^{n}$, the rest of parameters present in $\mathcal{U}_{d}^{n}$ manifesting themselves only through charged currents (and thus through neutrino oscillations). To achieve this goal, the chosen order of matrices $\mathcal{U}_{d}^{n}$ and $\mathcal{U}_{s}^{n}$ relative to $\mathcal{U}_{m}^{n}$ in eq. (42) is crucial. The factorization property of the charged and neutral currents makes the parametrization eq. (42) very convenient in practice. Altogether, the total neutrino mixing matrix $\mathcal{U}^{n}$ for the general $(d, s)$ extension contains $d(d-1) / 2+s d$ physical mixing angles and the same number of phases in agreement with refs. [2], [3]. Similarly to eqs. (44), (45) one can propose the following explicit representation for $\mathcal{U}_{m}^{n}$

$$
\mathcal{U}_{m}^{n}=\prod_{\substack{f=1, \ldots, d \\ g=1, \ldots, s}} \otimes u_{f, d+g}\left(\omega_{f, d+g}\right)
$$

with a fixed but a priori unspecified order of submatrices, and $\omega_{f, d+g}$ being $d s$ arbitrary complex numbers. When restricted to $2 \times 2$ complex plane the matrices $u_{f, d+g}$ are quite similar to those given by eq. (45). By means of the identity (46) with diagonal phases from $U_{s}^{\nu}$, one can eliminate $s$ phases out of $d s$ ones in $\mathcal{U}_{m}^{n}$, and to get in the end $d+s-1$ Majorana specific phases in $\mathcal{U}^{n}$.

As for the renormalizable $(d, s)_{\mathrm{r}}$ extensions, the $d \times d$ symmetric matrix constraint eq. (26) reduces $d(d+1) / 2$ phases and the same number of moduli, $d$ of the latter ones corresponding to masses and $d(d-1) / 2$ to mixing angles. As a result, $\mathcal{U}^{n}$ contains $s d$ independent physical mixing angles and $d(s-$ 1) phases, precisely as it should according to general counting of ref. [2] . Superficially, the above constraint restricts only parameters in $\mathcal{U}_{m}^{n}$ and does not touch those in $U_{d}^{\nu}$. But it can be shown that at $d \geq s>0$, due to the presence of $d-s$ massless neutrinos, it is additionally possible to eliminate from $U_{d}^{\nu}$ the parameters corresponding to $U(d-s)$. It leaves in $U_{d}^{\nu} d s-s(s+$

\footnotetext{
${ }^{15}$ This follows from inversion of eq. (11) for transformation between the weak and mass neutrino bases.
} 
1) $/ 2$ independent $\theta$ 's and $d(s-1)-s(s-1) / 2 \delta$ 's. Note that the constraint does not invalidate the charged-neutral current factorization property.

This gives the complete solution to the problem. There are two important cases with neutral currents remaining diagonal.

(ii) Only Dirac masses For the particular case of the $(d, s)_{\mathrm{r}}$ extension with only Dirac masses, further reduction of parameters is possible. Diagonalization of the neutrino mass matrix by $\mathcal{U}_{d}^{n} \mathcal{U}_{s}^{n}$ yields for the $d \times s$ Dirac mass term

$$
m_{\text {diag }}=U_{d}^{\nu T} m U_{s}^{N},
$$

with the non-negative elements on the quasi-diagonal, the rest being zero. Remind that $U_{s}^{N}$ is unobservable. At $0<s \leq d$ one has $s$ nonzero entries in $m_{\text {diag. }}$. Hence, there is the $U(d-s) \times U(1)^{s-1}$ left-out symmetry in the doublet neutrino subspace which reduces the number of parameters in $U_{d}^{\nu}$ to $s d-s(s+1) / 2$ mixing angles and $s d-s(s+1) / 2-d+1$ phases. At $0<d<s$ there are $d$ nonzero entries, the left-out symmetry in the doublet neutrino subspace is only $U(1)^{d-1}$ and one recovers the CKM-like scheme for $d$ Dirac neutrinos with $d(d-1) / 2$ mixing angles and $(d-1)(d-2) / 2$ phases. This explicit counting is in complete accordance with the general one in ref. [2].

Finally, there still remains the maximal (equal to $\pi / 4$ ) mixing $\mathcal{U}_{m}^{n}$ between the pairs of the mass degenerate eigenfields. Under the proper choice for $\varphi$, the ensuing orthogonal transformation $\mathcal{O}^{n}$ brings the neutrino mass matrix to the real diagonal form $\mathcal{M}_{\text {diag }}^{n}=\left(m_{1}(1,-1), \ldots, m_{p}(1,-1), 0, \ldots, 0\right)$, with $p=\min (d, s)$. It corresponds to $p$ pairs of the mass degenerate Majorana neutrinos with opposite $C P$ parities plus $|s-d|$ massless neutrinos. The emerging mixing matrix $X$ in eq. (50) is superficially non-diagonal. Nevertheless the neutral currents may be put to explicitly flavour conserving form independent of $\varphi$ via the reversed transition to the Dirac basis. As for massless neutrinos, there is no difference whether they are considered as Weyl or Majorana ones. The neutral current Lagrangian $\mathcal{L}_{Z}^{n}$ for the doublet massless neutrinos is flavour conserving, singlet massless neutrinos being sterile.

(iii) Only Majorana masses In the case of $(d, s)$ extension with only Majorana masses, one has $\mathcal{U}_{m}^{n} \equiv I$ and hence $X \equiv P^{n}$. The neutrino part of 
interactions now becomes

$$
\mathcal{L}_{Z}^{n}=\frac{g}{4 c_{W}} Z_{\alpha} \overline{\mathcal{N}} \gamma^{\alpha} \gamma_{5} P^{n} \mathcal{N}
$$

and (in the unitary gauge)

$$
-\mathcal{L}_{Y}^{n}=\left(\frac{H}{v}+\frac{1}{2}\left(\frac{H}{v}\right)^{2}\right) \overline{\mathcal{N}} \mathcal{M}_{\text {diag }}^{n} P^{n} \mathcal{N},
$$

both Lagrangians being explicitly independent of $\varphi$. Due to presence of the on-doublet neutrino projector $P^{n}$ the singlet neutrinos are insured to be sterile.

(iv) Small doublet-singlet mixing It is instructive to discuss the mixing matrices under condition of a small doublet-singlet mixing, the case of importance for phenomenology. In particular this is so in the framework of the see-saw approximation (see further on). Making use of the equivalent representation for eq. (51) as

$$
\mathcal{U}_{m}^{n}=\exp \left(\begin{array}{cc}
0 & \omega \\
-\omega^{\dagger} & 0
\end{array}\right)
$$

where $\omega$ is an arbitrary complex $d \times s$ matrix, one gets for small $\omega$

$$
\mathcal{U}_{m}^{n}=\left(\begin{array}{cc}
\left(1-\frac{1}{2} \omega \omega^{\dagger}\right) & \omega\left(1-\frac{1}{6} \omega^{\dagger} \omega\right) \\
-\omega^{\dagger}\left(1-\frac{1}{6} \omega \omega^{\dagger}\right) & 1-\frac{1}{2} \omega^{\dagger} \omega
\end{array}\right)+\mathcal{O}\left(\omega^{4}\right)
$$

and

$$
\mathcal{U}^{n}=\left(\begin{array}{cc}
U_{d}^{\nu}\left(1-\frac{1}{2} \omega \omega^{\dagger}\right) & U_{d}^{\nu} \omega\left(1-\frac{1}{6} \omega^{\dagger} \omega\right) \\
-\omega^{\dagger}\left(1-\frac{1}{6} \omega \omega^{\dagger}\right) & 1-\frac{1}{2} \omega^{\dagger} \omega
\end{array}\right)+\mathcal{O}\left(\omega^{4}\right) .
$$

Hence one has

$$
V=\left(U_{d}^{\nu}\left(1-\frac{1}{2} \omega \omega^{\dagger}\right) \quad U_{d}^{\nu} \omega\right)+\mathcal{O}\left(\omega^{3}\right)
$$

as well as

$$
X=\left(\begin{array}{cc}
1-\omega \omega^{\dagger} & \omega \\
\omega^{\dagger} & \omega^{\dagger} \omega
\end{array}\right)+\mathcal{O}\left(\omega^{3}\right)
$$


These expressions can readily be generalized with any finite accuracy in $\omega .16$

Finally, the constraint for the $(d, s)_{\mathrm{r}}$ extension given by eq. (25) yields

$$
\varphi^{\nu *} m_{\text {diag }}^{\nu}=-\omega \varphi^{N *} M_{\text {diag }}^{N} \omega^{T}+\mathcal{O}\left(\omega^{4}\right) .
$$

This determines $m_{\text {diag }}^{\nu}$ and a part of the $\omega$ 's in terms of $M^{N}$ and the rest of the $\omega$ 's. E.g., in the simplest case $s=1$ the solution to the equation can be shown to be given by the $d$-dimensional vector $\omega$ with one nonzero component $\omega_{d}=\left(-\varphi_{d}^{\nu} / \varphi^{N}\right)^{1 / 2}|\omega|$, so that $m_{d}=|\omega|^{2} M$. Reversing, one gets generically $\omega=\mathcal{O}\left(\left|m_{\text {diag }}^{\nu} / M_{\text {diag }}^{N}\right|^{1 / 2}\right)$. The general solution to eq. (60) is given by an $s \times s$ nonzero matrix with the proper constraints followed from the equation. As a result, the parameters in $\mathcal{U}^{n}$ are shared between the independent ones in $\mathcal{U}_{d}^{n}$ and $\mathcal{U}_{m}^{n}$ as is shown in Table 1 . The relations above have their close

Table 1 Independent mixing parameters for the renormalizable $(d, s)_{\mathrm{r}}$ extensions.

\begin{tabular}{|l|c|c|c|c|}
\hline & Param's & $\mathcal{U}^{n}$ & $\mathcal{U}_{d}^{n}$ & $\mathcal{U}_{m}^{n}$ \\
\hline \multirow{3}{*}{$d \geq s>0$} & Angles & $d s$ & $\begin{array}{c}d(d-1) / 2 \\
-(d-s)(d-s-1) / 2\end{array}$ & $s(s+1) / 2$ \\
\cline { 2 - 5 } & & & $\begin{array}{c}d(d-1) / 2 \\
-(d-s)(d-s+1) / 2\end{array}$ & $s(s-1) / 2$ \\
\hline$s>d>0$ & Ahases & $d(s-1)$ & $d(d-1) / 2$ & $s d-d(d-1) / 2$ \\
\cline { 2 - 5 } & & & $d(d-1) / 2$ & $s d-d(d+1) / 2$ \\
\hline \multirow{2}{*}{$d=s=n$} & Phases & $d(s-1)$ & $n(n-1) / 2$ & $n(n+1) / 2$ \\
\cline { 2 - 5 } & Angles & $n^{2}$ & $n(n-1) / 2$ & $n(n-1) / 2$ \\
\hline
\end{tabular}

counterparts in the framework of the see-saw approximation (see below).

The part $\left.\mathcal{U}^{n}\right|_{d \times d}$ of the total mixing matrix $\mathcal{U}^{n}$ which spans the $d \times d$ subspace of the doublet neutrinos reads

$$
\left.\mathcal{U}^{n}\right|_{d \times d}=U_{d}^{\nu}\left(1-\frac{1}{2} \omega \omega^{\dagger}\right)+\mathcal{O}\left(\omega^{4}\right)
$$

\footnotetext{
${ }^{16}$ Clearly, the above results are not applicable in the case of the pseudo-Dirac neutrinos where $\omega$ 's are generally not small. Here eq. (55) could be properly modified by decomposing mixing matrix $\mathcal{U}_{m}^{n}$ into the product of two parts, $\mathcal{U}_{m}^{n} \equiv \mathcal{U}_{m 2}^{n} \mathcal{U}_{m 1}^{n}$. The part $\mathcal{U}_{m 1}^{n}$ should produce transition to the pseudo-Dirac basis by a set of the (mutually commuting) pairwise transformations at the (nearly) $\pi / 4$ angles. The part $\mathcal{U}_{m 2}^{n}$ due to the rest of $\omega$ 's could result in the remaining flavour violating corrections.
} 
It includes the $d \times d$ Hermitian combination $\omega \omega^{\dagger}$ of the $d \times s$ matrix $\omega$. This brings in the additional mixing angles and phases. But even in neglect of these terms, when $\left.\mathcal{U}^{n}\right|_{d \times d}=U_{d}^{\nu}$ is unitary, the number of physical phases in it being relevant for the Majorana neutrinos, $d(d-1) / 2$, would exceed that $(d-1)(d-2) / 2$ given by the CKM-like unitary matrix for the Dirac neutrinos. In essence, this difference is due to having the freedom of fixing in $U_{d}^{\nu}$, out of the initial $d(d+1) / 2$ phases, only $d$ phases in the Majorana case, instead of $2 d-1$ ones in the Dirac case.

(v) See-saw approximation In order to evaluate the mixing magnitudes and study the decoupling limit, it is useful to compare the general results for small mixing with those obtained in the framework of the see-saw mechanism by the explicit diagonalization of the neutrino mass matrix. By the unitary global transformation $U(s)$ of the singlet neutrinos the mass matrix $M$ in eq. (7) can be put to the diagonal form

$$
M=\varphi^{N} M_{\text {diag }}^{N} .
$$

Besides, $d$ phases of the Dirac mass matrix $m$ can be eliminated due to the freedom of the charged lepton phase redefinitions. This freedom is still left after the simultaneous diagonalization of the charged lepton mass matrix by the bi-unitary $d \times d$ transformation. So, the total neutrino mass matrix $\mathcal{M}^{n}$ clearly contains $s(d+1)$ independent moduli, $s$ of them corresponding to physical masses and $s d$ ones to mixing angles, as well as $d(s-1)$ phases. This explicit counting for the $(d, s)_{\mathrm{r}}$ extension is in accordance with the general one presented in ref. [2].

The results of ref. 12 for the neutrino mass diagonalization in the $(n, n)_{\mathrm{r}}$ extension can readily be generalized to the $(d, s)_{\mathrm{r}}$ one. Under condition $M_{\text {diag }}^{N} \gg|m|$ for all the elements, the see-saw neutrino mixing matrix can be found to be

$$
\mathcal{U}_{m}^{n^{\prime}}=\left(\begin{array}{cc}
1-\frac{1}{2} \xi^{\dagger} \xi & \xi^{\dagger}\left(1-\frac{1}{2} \xi \xi^{\dagger}\right) \\
-\xi\left(1-\frac{1}{2} \xi^{\dagger} \xi\right) & 1-\frac{1}{2} \xi \xi^{\dagger}
\end{array}\right)+\mathcal{O}\left(\xi^{4}\right)
$$

where the $s \times d$ matrix $\xi$ is $\xi \equiv M^{-1} m^{T},|\xi| \ll 1$. Clearly, $\xi$ results in $s d$ mixing angles and $d(s-1)$ phases in the neutrino mixing matrix. Up to next-to-leading order in $\xi$ the matrix $\mathcal{U}_{m}^{n^{\prime}}$ brings $\mathcal{M}_{0}^{n}$ from the texture form

$$
\mathcal{M}_{0}^{n}=\left(\begin{array}{cc}
0 & \xi^{T} M \\
M \xi & M
\end{array}\right)
$$


to the block-diagonal form $\mathcal{M}^{n^{\prime}}=\mathcal{U}_{m}^{n^{\prime} T} \mathcal{M}_{0}^{n} \mathcal{U}_{m}^{n^{\prime}}$ with

$$
\mathcal{M}^{n^{\prime}}=\left(\begin{array}{cc}
-\xi^{T} M \xi & 0 \\
0 & M+\frac{1}{2}\left(M \xi \xi^{\dagger}+\xi^{*} \xi^{T} M\right)
\end{array}\right)+\mathcal{O}\left(\xi^{3}\right) .
$$

Now, by means of the unitary $d \times d$ transformation $U_{d}^{\nu^{\prime}}$ one can diagonalize the mass matrix for light neutrinos

$$
\varphi^{\nu} m_{\mathrm{diag}}^{\nu}=-U_{d}^{\nu^{\prime}} \xi^{T} \varphi^{N} M_{\mathrm{diag}}^{N} \xi U_{d}^{\nu^{\prime}}+\mathcal{O}\left(\xi^{4}\right)
$$

so that $\xi=\mathcal{O}\left(\left(m^{\nu} / M^{N}\right)^{1 / 2}\right)$. Similarly, by the unitary $s \times s$ transformation $U_{s}^{N^{\prime}}=I_{s}+\mathcal{O}\left(\xi^{2}\right)$ one can diagonalize the mass matrix for the heavy neutrinos. Under condition that the left-handed Majorana mass term $\mu$ is $\mathcal{O}(1 / M)$, eq. (66) straightforwardly generalizes to

$$
\varphi^{\nu} m_{\mathrm{diag}}^{\nu}=U_{d}^{\nu^{\prime}}\left(\mu-\xi^{T} \varphi^{N} M_{\mathrm{diag}}^{N} \xi\right) U_{d}^{\nu^{\prime}}+\mathcal{O}\left(1 / M^{3}\right) .
$$

The full neutrino mixing matrix in the see-saw framework looks like $\mathcal{U}^{n}=$ $\mathcal{U}_{m}^{n^{\prime}} \mathcal{U}_{d}^{n^{\prime}} \mathcal{U}_{s}^{n^{\prime}}$. Comparing it with that in the the doublet-singlet parametrization eq. (42), one finds that the parametrizations differ by order of matrices. As a result, this leads to somewhat different representations for $V$ (and $X$ ). In the absence of the direct masses for the doublet neutrinos the modelindependent matrix $\omega$ is related with the see-saw one $\xi$ as

$$
\xi=\left(U_{d}^{\nu} \omega\right)^{\dagger}+\mathcal{O}\left(\omega^{3}\right)
$$

where $U_{d}^{\nu^{\prime}}=U_{d}^{\nu}+\mathcal{O}\left(\omega^{2}\right)$. In this, all the quantities $\omega, U_{d}^{\nu}$ and $\xi$ generally depend on $\varphi$. The parameters of $\xi$ are clearly shared between the independent ones in $U_{d}^{\nu}$ and $\omega$ in accordance with Table 1.

In the limit $\xi=\mathcal{O}(m / M) \rightarrow 0$ and hence $m^{\nu} \rightarrow 0$, one has to substitute effectively $U_{d}^{\nu} \rightarrow I_{d}$ due to the neutrino mass degeneracy. So, all the light neutrino mixing effects in the see-saw framework disappear at $v / M \ll 1$ signalling the onset of decoupling. In particular, it follows from eqs. (24), (27) that Higgs boson decouples from the $\nu N$ current in the see-saw framework in the leading order $\mathcal{O}(M)$, only Yukawa couplings $\mathcal{O}(v)$ being generally left. As for $N N H$ vertices, they are $\mathcal{O}\left(v^{2} / M\right)$ in the limit $M \gg v .17$ The see-saw

\footnotetext{
${ }^{17}$ This contradicts the statement of ref. [4 made in the see-saw framework on significant enhancement of the $\nu N H$ and $N N H$ vertices. The enhancement could clearly take place at large $M$ only in neglect of the suppression $\left(\sim 1 / M\right.$ or $\left.1 / M^{2}\right)$ of the mixing elements. Otherwise it could be just a numerical effect at not too large $M$.
} 
matrix $\xi$ (and more generally $\omega$ ) results in the non-universality and nonunitarity of the lepton charged and neutral currents, and it can be estimated experimentally to be small, typically $|\xi| \leq \mathcal{O}\left(10^{-1} \div 10^{-2}\right)[13]$.

Some comments are finally in order. It is clear from the above that the see-saw form of $\mathcal{U}_{m}^{n^{\prime}}$, given by eq. (63), closely resembles the most general one given by eq. (56). In fact, this see-saw-like structure does not depend on the particular expression eq. (7) for the neutrino mass matrix, the latter restricting only the number of independent parameters through constraint eq. (60). Whereas the see-saw results, under condition $m^{\nu} \neq 0$, can strictly be applicable only at $\xi \neq 0$, the advantage of the model-independent parametrization is that it can straightforwardly be generalized to a case with arbitrary $\omega$. The mixings and masses become completely disentangled. In particular, one can have, e.g., $\omega=0$ at $m^{\nu} \neq 0$, or $m^{\nu}=0$ at $\omega \neq 0$. Besides, it is possible to have finite $\omega$ at $M \gg v$ and thus produce enhancement in the vertices with heavy neutrinos 18 This general parametrization completely exhausts all the possibilities for the neutrino masses, including these of the pure Dirac and Majorana origins.

\section{$5 \quad$ Neutrino oscillations}

The structure of $\mathcal{U}^{n}$ for the SM general extension $(d, s)$ (in practice, $d=3$ ) could be used when discussing the pattern of the light neutrino oscillations. Both Dirac and Majorana light neutrinos are permitted a priori. Because the Dirac neutrino can be regarded as a pair of the mass degenerate Majorana ones (with opposite CP parities), the SM extensions with at least several additional light degrees of freedom are of interest. The primordial abundances of light nuclei in the standard big bang nucleosynthesis restrict the effective number of the relativistic two component interacting neutrinos to be $<3.2$ (95\% C.L.) [14]. Hence, in principle, a number of the light sterile neutrinos could still be accommodated. Sticking to as simple neutrino content as possible one can encounter two different scenarios: with and without one additional light Majorana neutrino.

\footnotetext{
${ }^{18}$ Clearly, the violation of decoupling can originate in the given framework only due to non-renormalizable Lagrangian (2).
} 
(i) No light singlet neutrino The relevant for oscillations part of the neutrino mixing matrix $\mathcal{U}^{n}$ at any $s$ reduces in this case to $\left.\mathcal{U}^{n}\right|_{d \times d}$. In the leading $\mathcal{O}(\omega)$ approximation it is $d \times d$ unitary matrix $U_{d}^{\nu}$. This effectively simplifies the $(d, s)$ extension up to $(d, 0)$ in the light lepton sector (in practice, it is $(3,0)$ one and the corresponding mixing matrix is given by eq. (48)). As is stated before, $U_{d}^{\nu}$ depends generally on $d(d-1) / 2$ physical mixing angles and the same number of phases. But, according to eq. (29), the neutrino oscillations with chirality conservation (coinciding here with the total lepton number conservation, $\Delta L=0$ ) are insensitive to $d-1$ phases capable of being resided in the Majorana condition matrix $\varphi$. This reduces the number of observable phases to $(d-1)(d-2) / 2$, exactly as in the Dirac case. Hence, there is no difference here for the $\Delta L=0$ neutrino oscillations between the Majorana and Dirac cases [15]. This effective suppression could be evaded though for the chirality flipping (here also lepton number violating, $|\Delta L|=2$ ) oscillations. But according to eq. (30) these ones are, in their turn, chirally suppressed, i.e., their intensity is $\mathcal{O}\left(\left(m^{\nu} / E\right)^{2}\right)$ at the neutrino energy $E>m^{\nu}$ [15, 16]. It follows that it would be hard in this case to observe in oscillations the Majorana specific $C P$ violation, if any.

Finally, in the absence of light singlet neutrinos the chirality preserving light neutrino oscillations are described in the given assumptions just by the $d \times d$ unitary matrix $U_{d}^{\nu}$ of the CKM-like type with $d(d-1) / 2$ mixing angles and $(d-1)(d-2) / 2$ phases. Account for the terms $\mathcal{O}\left(\omega^{2}\right)$ due to the doublet-singlet mixing would reveal additional $C P$ violating phases in $U_{d}^{\nu}$ (plus those in $\omega$ itself). Besides, it is clear that the neutrino oscillations in this case are mainly sensitive to other set of the mixing parameters than the neutral current mixing matrix, the latter one being determined entirely by the doublet-singlet mixing matrix $\mathcal{U}_{m}^{n}(\omega)$. Hence in this case the two phenomena disentangle in essence.

(ii) Light singlet neutrino As for the case with a light singlet neutrino, the doublet-singlet mixing can no more be ignored and should be taken into account, producing the observable effect. Among singlet neutrinos, only the light one is relevant in the leading order $\mathcal{O}(\omega)$ for the light neutrino mixing. One can effectively put in the given approximation $s=1$, thus reducing the problem to the $(d, 1)$ case. $\mathcal{U}_{m}^{n}$ in eq. (56) is given by its part not higher than $\mathcal{O}(\omega)$, where $\omega=\left(\omega_{1}, \ldots, \omega_{d}\right)$. Thus, $\mathcal{U}^{n}$ effectively depends on $d(d+$ 
1)/2 physical mixing angles and equal number of phases, in accordance with ref. [2]. $d(d-1) / 2$ of each of them reside in the doublet-doublet mixing $U_{d}^{\nu}$ and $d$ in the doublet-singlet mixing $\mathcal{U}_{m}^{n}(\omega)$. Such an approximate $(d+1) \times(d+1)$ matrix $\mathcal{U}^{n}$ is unitary up to the given accuracy and presents the most general mixing matrix in this approximation. Due to explicit independence on $\varphi$ of the helicity conserving (now do not coinciding any more with lepton number conserving) oscillations the number of phases relevant to these oscillations reduces to $d(d-1) / 2$, as if there were $d+1$ Dirac neutrinos.

In reality, one has $d=3$ and experiment might suggest a pairwise neutrino mixing [1]. It consists only of the mixing of a pair of doublet neutrinos (chosen here as $\nu_{1}$ and $\nu_{2}$ ) between themselves and the mixing of the light singlet neutrino $\left(N_{1} \equiv \nu_{4}\right)$ only with the remaining doublet neutrino $\nu_{3}$, i.e., $\omega=\left(0,0, \omega_{3}\right)$. Hence, in the case at hand the neutrino mixing $(3,1)$ reduces to the product of two cases $(2,0)$ and $(1,1)$, each of them corresponding to one mixing angle and one Majorana specific phase (the latter being unobservable in the helicity conserving oscillations). Under proper redefinitions, the mixing matrix $\mathcal{U}^{n}$ becomes

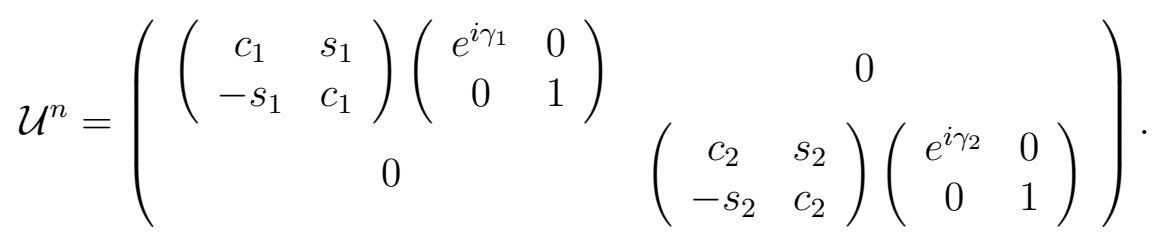

In the given assumptions, eq. (69) describes the general, consistent with experiment, mixing for four light neutrinos, one of them being (quasi-)sterile. Correspondingly, one gets for the charged current mixing matrix $V=P^{e n} \mathcal{U}^{n}$

$$
V=\left(\begin{array}{cccc}
c_{1} & s_{1} & 0 & 0 \\
-s_{1} & c_{1} & 0 & 0 \\
0 & 0 & c_{2} & s_{2}
\end{array}\right)\left(\begin{array}{cccc}
e^{i \gamma_{1}} & 0 & 0 & 0 \\
0 & 1 & 0 & 0 \\
0 & 0 & e^{i \gamma_{2}} & 0 \\
0 & 0 & 0 & 1
\end{array}\right)
$$

and for the neutral current one $X=\mathcal{U}^{n} P^{n} \mathcal{U}^{n \dagger}=V^{\dagger} V$

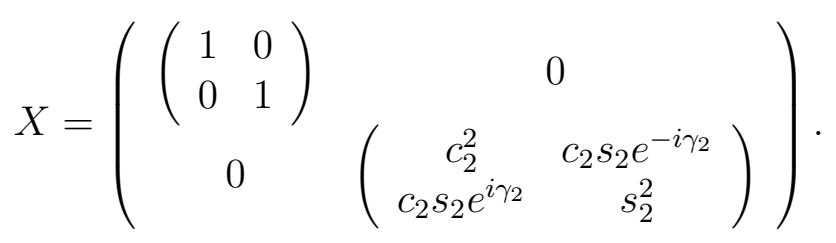


Clearly, $c_{2}, s_{2} \neq 0$ results in flavour violation in neutral currents. Remind that to completely describe the lepton interactions one should also specify

the matrix $\eta_{C P}$ of the neutrino $C P$ signatures, as well as the matrix $\varphi$ of Majorana condition to which the mixing matrices above correspond. In particular, only then one can decide whether there is $C P$ violation or not in a general case. But in the chirality preserving oscillations, CP will always be conserved because all the phases here are the Majorana specific ones.

\section{Summary}

The neutrino gauge and Yukawa interactions for the SM extensions, both renormalizable and effective, are systematically investigated under arbitrary Majorana condition. Independence of the particular choice of this condition is demonstrated by means of the explicit rephasing invariance. The invariance is used to exhibit manifestations of the Majorana specific phases. The parametrization of the neutrino mixing matrices in the doublet-singlet factorized form is proposed. Its relation with the see-saw approximation is shown. The patterns of neutrino mixing, relevant to neutrino oscillation experiments, are exposed.

The author is grateful to V.V. Kabachenko for valuable discussions.

\section{References}

[1] S.M. Bilenky et al., Summary of the NOW'98 Phenomenology Working Group, hep-ph/9906251.

[2] Yu.F. Pirogov, Preprint IHEP 2000-2 (2000), hep-ph/0002299.

[3] J. Schechter and J.W.F. Valle, Phys. Rev. D22 (1980) 2227.

[4] A. Pilaftsis, Z. Phys. C55 (1992) 275, hep-ph/9901206.

[5] T. Yanagida, Prog. Theor. Phys. B135 (1978) 66; in Proc. of the Workshop on Unified Theory and Baryon Number of the Universe, eds. O. Swada and A. Sugamoto (KEK, 1979) p. 95; M. Gell-Mann, P. Ramond and R. Slansky, in Supergravity, eds. P. van Nieuwenhuisen ans D. Freedman (North-Holland, Amsterdam, 1979) p. 315. 
[6] Yu.F. Pirogov and O.V. Zenin, Eur. Phys. J. C10 (1999) 629, hepph/9808396.

[7] J. Bernabeu and P. Pascual, Nucl. Phys. B228 (1983) 21; A. Barroso and J. Maalampi, Phys. Lett. 132B (1983) 355; P.J. O'Donnell and U. Sarkar, Phys. Rev. D52 (1995) 1720, hep-ph/9305338.

[8] B. Kaiser, Phys. Rev. D30 (1984) 1023.

[9] S.M. Bilenky, N.P. Nedelcheva and S.T. Petcov, Nucl. Phys. B247 (1984) 61.

[10] C. Giunti, C.W. Kim and U.W. Lee, Phys. Rev. D44 (1991) 3635, ibid. D45 (1992) 2414; Phys. Lett. 421B (1998) 237, hep-ph/9709494; C. Giunti, C.W. Kim, J.A. Lee and U.W. Lee, Phys. Rev. D48 (1993) 4310, hep-ph/9305276.

[11] L. Wolfenstein, Phys. Lett. 107B (1981) 77; J. Schechter and J.W.F. Valle, Phys. Rev. D24 (1981) 1883; Err. ibid. D25 (1982) 283.

[12] W. Buchmüller and D. Wyler, Phys. Lett. 249B (1990) 458; W. Buchmüller and C. Greub, Phys. Lett. 256B (1991) 465; Nucl. Phys B363 (1991) 365 .

[13] M. Gronau, C.N. Leung and J.L. Rosner, Phys. Rev. D29 (1984) 2539; P. Langacker and D. London, Phys. Rev. D38 (1988) 886, 907; S.M. Bilenky, W. Grimus and H. Neufeld, Phys. Lett. 252B (1990) 119.

[14] D. Tytler, J.M. O’Meara, N. Suzuki and D. Lubin, astro-ph/0001318.

[15] J. Schechter and J.W.F. Valle, Phys. Rev. D23 (1981) 1666; M. Doi, T. Kotani, H. Nishiura, K. Okuda and E. Takasugi, Phys. Lett. 102B (1981) 323.

[16] J. Bahcall and H. Primakoff, Phys. Rev. D18 (1978) 3463. 